\title{
NEUROPSIQUIATRIA: PET Y SPECT
}

\section{Dr. Juan Carlos Quintana F.}

Medicina Nuclear. Pontificia Universidad Católica de Chile

Abstract: Functional brain imaging with PET and SPECT have a definitive and well established role in the investigation of a variety of conditions such as dementia, epilepsy and drug addiction. With these methods it is possible to detect early rCBF (regional Cerebral Blood Flow) changes seen in dementia (even before clinical symptoms) and differentiate Alzheimer's disease from other dementias by means of the rCBF pattern change. 18-F-FDG PET imaging is a useful tool in partial epilepsy because both rCBF and brain metabolism are compromised at the epileptogenic focus. During the seizure, $\mathrm{rCBF}$ dramatically increases locally. Using SPECT it is possible to locate such foci with $97 \%$ accuracy. In drug addiction, particularly with cocaine, functional imaging has proven to be very sensitive to detect brain flow and metabolism derangement early in the course of this condition. These findings are important in many ways: prognostic value, they are used as a powerful reinforcement tool and to monitor functional recovery with rehabilitation. There are many other conditions in which functional brain imaging is of importance such as acute stroke treatment assessment, trauma rehabilitation and in psychiatric and abnormal movement diseases specially with the development of receptor imaging.

Key words: Functional brain imaging, SPECT, 18F-FDG PET, Dementia, Alzheimer's disease, Epilepsy, Cocaine, Mood and movement disorders.

Resumen: Existen numerosas indicaciones claramente establecidas para el uso del SPECT y PET en patología neuro-psiquiátrica, particularmente en el estudio de demencias, epilepsia y adicción a drogas. Estos métodos permiten detectar precozmente

Quintana JC. Neuropsiquiatría: PET y SPECT. Rev Chil Radiol 2002; 8:63-69.

Correspondencia: Dr. Juan C. Quintana F.

Marcoleta 347, Santiago CP: 6510260

jcquinta@med.puc.cl (aun antes de las manifestaciones clínicas) cambios en la perfusión y metabolismo cerebral en pacientes con demencias. Es posible además diferenciar la enfermedad de Alzheimer de otras causas de demencia, analizando el patrón de la alteración neurofuncional. En epilepsia parcial, tanto el metabolismo como la perfusión están alterados en el foco epileptogénico, lo que puede ser detectado con F-18 FDG PET. Durante la crisis epiléptica, el flujo sanguíneo puede aumentar dramáticamente en el foco epileptogénico, lo que puede ser detectado con SPECT con $97 \%$ de certeza. En pacientes drogadictos, especialmente a la cocaína, estos métodos han demostrado ser muy sensibles para la detección precoz de cambios en el flujo y metabolismo cerebral, lo que es clínicamente importante en varios aspectos: 1) Tiene valor pronóstico (neuro-funcional), 2) Se puede usar para aumentar la adherencia a la terapia y 3) Permite evaluar objetivamente la recuperación funcional. Existen muchas otras indicaciones presentes y futuras, por ejemplo: en la monitorización de la revascularización en accidentes vasculares cerebrales agudos, rehabilitación post TEC, estudio de patología psiquiátrica y movimientos anormales especialmente con el desarrollo de radioligandos.

Palabras claves: Imágenes funcionales de cerebro, SPECT, F-18-FDG PET, Demencia, Enfermedad de Alzheimer, Epilepsia, cocaína, Trastornos del ánimo y movimientos anormales.

\section{Introducción}

Los avances tecnológicos experimentados en el campo de las imágenes funcionales y su mayor disponibilidad han permitido extender sus indicaciones y sus usos en patología neuropsiquiátrica. Particularmente el uso del F-18 FDG PET se ha incrementado enormemente desde que la FDA (Food and Drug Administration) aprobara su uso en la práctica clínica en $1997^{(1)}$. En este artículo se hará mención a las indicaciones más comunes tanto de SPECT como PET en patología neuropsiquiátrica con énfasis en el los estudios de perfusión cerebral y análisis metabólicos con F-18-FDG PET. 


\section{Envejecimiento Normal}

Numerosos autores sugieren que existe una disminución del metabolismo cerebral de la glucosa con la edad. Sin embargo, se han observado algunas inconsistencias entre los diferentes estudios con respecto a las áreas más afectadas. Algunos autores han observado mayor compromiso de la región frontal y corteza somato-sensorial ${ }^{(2,3)}$ y otros sugieren además una disminución de la actividad metabólica temporal con la edad. También se ha observado que existen diferencias entre hombres y mujeres ${ }^{(4)}$. En contraposición a lo anterior, otros autores ${ }^{(5)}$ no encontraron diferencias significativas entre el metabolismo de la glucosa a diferentes edades o entre hombres y mujeres, cuando los datos fueron corregidos por el grado de atrofia cortical.

\section{Enfermedad de Alzheimer}

El criterio clínico para el diagnóstico de la enfermedad de Alzheimer fue definido en 1984 por el NINCDS-ADRDA ${ }^{(6)}$ (National Institute of Neurological and Communicative Disorders and Stroke Alzheimer's Disease and Related Disorders). En él se establece que para el diagnóstico de la enfermedad se requiere demostrar la existencia de deterioro cognitivo crónico y progresivo en pacientes adultos o ancianos, sin otras causas subyacentes que puedan explicar este fenómeno. El problema es que, usando este criterio, es difícil diferenciar entre enfermedad de Alzheimer y otras causas de deterioro en etapas tempranas de la enfermedad. Las imágenes funcionales de cerebro tendrían un rol en este sentido para establecer el diagnóstico diferencial y ayudar a entender los mecanismos subyacentes de la enfermedad ${ }^{(4)}$

En los primeros estudios con PET en pacientes con enfermedad de Alzheimer, se observó que éstos presentaban una disminución global del metabolismo de la glucosa del orden de 20 a 30\% con relación a los controles de edad equivalente ${ }^{(4)}$. En otros estudios se observó además del hipometabolismo global, un mayor compromiso bilateral de la región temporo-parietal ${ }^{(7)}$. Esto es lo que se denomina el patrón típico de la enfermedad de Alzheimer y puede ser especialmente pronunciado en pacientes menores de 65 años. Este patrón es altamente predictivo de esta enfermedad, sin embargo, no es patognomónico: puede verse en enfermedad de Parkinson, hematoma subdural parietal bilateral, infarto parietal bilateral y radioterapia parietal bilateral $^{(8)}$. El patrón típico antes descrito es similar tanto para SPECT como para PET, pero con éste último método la sensibilidad y la especificidad sería mayor especialmente en las etapas tempranas de la enfermedad, probablemente por un mayor contraste entre la captación de la corteza sensoriomotora versus la temporo-parietal ${ }^{(9)}$. Sin embargo, en un estudio longitudinal de 54 pacientes donde se comparó los hallazgos de perfusión cerebral con SPECT y la anatomía patológica, se observó que la sensibilidad y la especificidad del SPECT para la enfermedad de Alzheimer es de $86 \%$ y $73 \%$ respectivamente ${ }^{(10)}$ (Figura 1).

La extensión y magnitud de la alteración metabólica y de perfusión se correlaciona bien con la gravedad de los síntomas de la enfermedad(11). En pacientes con enfermedad inicial, existe disminución mínima del metabolismo de la glucosa y de la perfusión en la región parietal, conforme la enfermedad avanza se comienza a comprometer el metabolismo y la perfusión en la región frontal media izquierda y la región temporal alta bilateral. En pacientes con enfermedad avanzada, se observa hipometabolismo e hipoperfusión en las mismas regiones pero de mucho mayor intensidad. En todos los pacientes se observa que los lóbulos parietales son los más afectados con $38 \%$ de disminución del metabolismo en enfermedad moderada y $53 \%$ en enfermedad avanzada. El metabolismo y perfusión de otras áreas como la corteza visual y sensorio-motora, núcleos subcorticales, troncoencéfalo y cerebelo generalmente está preservado(4).

Las indicaciones clínicas para realizar SPECT y PET incluyen: confirmación del diagnóstico probable o diagnóstico posible de enfermedad de Alzheimer; diagnóstico diferencial con otros tipos de demencia como la Enfermedad Difusa por Cuerpos de Lewy, demencia vascular, demencia frontotemporal, enfermedad de Huntington, etc. Los hallazgos del SPECT tienen además valor pronóstico: la captación de l-123 iodoanfetamina (IMP) de la región parietal posterior derecha se correlaciona directamente con la sobrevida en pacientes con enfermedad de Alzheimer ${ }^{(12)}$.

\section{Enfermedad Difusa por Cuerpos de Lewy}

La enfermedad difusa por cuerpos de Lewy ha crecido en importancia en el último tiempo y se ha reportado ser la segunda causa de demencia después de la enfermedad de Alzheimer, representa hasta un $20 \%$ de todos los casos de demencia en la autopsia. Clínicamente se manifiesta como alucinaciones visuales, compromiso cognitivo fluctuante y síntomas parkinsonianos. En el comienzo de la enfermedad, la diferenciación con otros tipos de demencia puede ser difícil por sobreposición de síntomas. Existen evidencias que en esta enfermedad el compromiso del sistema colinérgico es mayor que en otras entidades, por lo que estos pacientes se beneficiarían mayormente con la terapia colinérgica.

Estudios realizados con SPECT sugieren que existe un patrón de hipoperfusión temporo-parietal bilateral similar a la enfermedad de Alzheimer, pero acompañado de hipoperfusión occipital(13). Esto ha 
sido reproducido en estudios realizados con SPM (Statistical Parametric Mapping) donde se comparó pacientes con enfermedad difusa por cuerpos de Lewy con pacientes con enfermedad de Alzheimer ${ }^{(14)}$. Utilizando estudios metabólicos con F-18 FDG PET, se encontró que los pacientes con enfermedad difusa por cuerpos de Lewy y los pacientes con Alzheimer presentaban una disminución global del metabolismo de la glucosa en relación a los normales y que los pacientes con enfermedad difusa por cuerpos de Lewy presentaban un compromiso significativamente mayor del metabolismo del lóbulo occipital(15).

\section{Demencia Fronto-Temporal}

Como su nombre lo indica, este es un grupo de enfermedades degenerativas que comprometen preferentemente los lóbulos frontales y temporales. La entidad se manifiesta con déficit cognitivo, de lenguaje y alteraciones del comportamiento. Inicialmente la memoria es encuentra preservada. Los hallazgos en el PET y SPECT incluyen hipometabolismo de la glucosa e hipoperfusión en los lóbulos frontales y la porción anterior de ambos lóbulos temporales. En estudios realizados con grandes grupos de pacientes, se ha podido demostrar que este patrón permiten diferenciar estos pacientes de otros tipos de demencia, como la enfermedad de Alzheimer ${ }^{(4,16)}$.

\section{Enfermedad de Parkinson}

Es un proceso degenerativo caracterizado por la pérdida de neuronas pigmentadas de la sustancia nigra. Esto produce una tríada sintomática: bradiquinesia, temblor y rigidez. Esta pérdida neuronal se asocia a disminución de la producción y almacenamiento de dopamina y disfunción del sistema nigro-estriatal. En $20-30 \%$ de los casos puede haber compromiso cognitivo. En los pacientes no tratados, se observa un aumento del metabolismo de la glucosa de los ganglios basales, que cuando los síntomas son unilaterales compromete a los ganglios contralaterales al lado afectado. El tratamiento con L-dopa no modifica las alteraciones metabólicas locales o globales. En aquellos pacientes con compromiso cognitivo se observa hipometabolismo cortical difuso. Sin embargo, en pacientes con demencia avanzada, se puede observar un patrón de perfusión indistinguible de la enfermedad de Alzheimer con hipometabolismo parieto-temporal y frontal bilateral.

Actualmente existen radioligandos para PET para receptores de Dopamina tanto pre-sinápticos (F18 Fluorodopa) como postsináptico (F-18 Nmetilespiperona). Esto permitirá realizar estudios más específicos en el diagnóstico, seguimiento y tratamiento de estos pacientes ${ }^{(16)}$.

\section{Enfermedad de Huntington}

La enfermedad de Huntington se presenta en la edad media de la vida, es de carácter autosómico dominante y se caracteriza por anormalidades motoras progresivas como movimientos coreiformes involuntarios y rigidez akinética ${ }^{(17)}$. Muchos pacientes sufren de cambios conductuales y deterioro cognitivo progresivo, llegando a síntomas de demencia. Los estudios de PET han demostrado hipometabolismo del núcleo caudado y putamen, cambios que preceden la atrofia demostrable por tomografía computada. Estos cambios metabólicos son más precoces en el núcleo caudado y se correlacionan con la capacidad funcional global del paciente mientras que el grado de hipometabolismo del putamen se correlaciona con la gravedad de las alteraciones motoras. Los pacientes en riesgo de la enfermedad presentan hipometabolismo del núcleo caudado y esto es concordante en $91 \%$ de los casos con los estudios genéticos ${ }^{(4)}$.

\section{Parálisis Supranuclear Progresiva}

Es una condición caracterizada por parálisis de la mirada, distonía, rigidez axial y eventualmente demencia. Esta enfermedad se asocia con disminución del metabolismo de la glucosa en los ganglios basales, tálamo, protuberancia y algunas áreas de la corteza cerebral. El metabolismo cerebeloso usualmente está preservado. El grado de compromiso cognitivo se correlaciona con el grado de compromiso de metabolismo cortical frontal $\left.\right|^{(4)}$.

\section{Epilepsia}

Esta enfermedad afecta entre 0,5 a $1 \%$ de la población, puede ser focal o generalizada y usualmente comienza en la niñez. En general durante la crisis el metabolismo y la perfusión cerebral aumentan considerablemente. El foco epileptogénico en la epilepsia parcial puede ser identificado porque durante el período interictal presenta disminución del metabolismo y generalmente disminución de la perfusión y durante el período crítico, hipermetabolismo e hiperperfusión. Durante el período intercrítico se puede localizar un foco único de hipometabolismo entre el 55 y $80 \%$ de los casos con alteraciones focales en el EEG. Sin embargo, existen algunos factores confundentes que deben ser tomados en consideración, como tipo de epilepsia, tiempo transcurrido desde el último episodio y tratamiento medicamentoso. Utilizando SPECT, la sensibilidad es menor, aproximadamente $50 \%$ de los casos presentan hipoperfusión interictal. Esto se explica porque se ha observado que en pacientes con epilepsia parcial existe un desacoplamiento entre la perfusión y el consumo de glucosa en el foco epileptogénico durante este periodo. La asimetría metabólica es mucho más marcada que la de perfusión en la región afectada.

Durante el período ictal la perfusión cerebral 
regional pueden aumentar hasta $300 \%$. Este fenómeno hace posible detectar el foco epileptogénico mediante el uso de técnicas como el SPECT, gracias a las características farmacocinéticas de los radiotrazadores utilizados. Sin embargo, prácticamente no existen estudios metabólicos con PET durante el período ictal debido a la complejidad de los mismos y la necesidad de tener al paciente en el equipo durante el episodio convulsivo. Un estudio ictal óptimo requiere que la administración del radiofármaco sea durante la crisis o en los 30 primeros segundos después de haber finalizado; entre uno y diez minutos después se denomina SPECT postictal. En grandes series de pacientes, la correcta identificación del foco epileptogénico utilizando SPECT ictal se logró en más del $90 \%$ de los casos, con lateralización incorrecta en $2 \%$. En estudios postictales (promedio 4 minutos post crisis), la lateralización correcta fue en $70 \%$ e incorrecta en $2 \%$.

La localización del foco epileptogénico es importante porque la remoción quirúrgica del mismo es un tratamiento efectivo para aquellos pacientes refractarios al tratamiento médico.

Otro aspecto interesante es predecir cuales pacientes tendrán un mejor o peor pronóstico después de la cirugía. Al respecto hay estudios que muestran que aquellos pacientes con hipometabolismo en el lóbulo temporal afectado tienden a tener mejor pronóstico y que mientras mayor es el grado de hipometabolismo, mejor es el resultado post-quirúrgico. Por el contrario, aquellos que tienen focos contralaterales y focos extratemporales tienen mayor probabilidad de tener recurrencias. La perfusión del tálamo también parece tener una importancia pronóstica, en el sentido que los pacientes que presentan hipometabolismo talámico contralateral al lóbulo afectado, tendrían un alto riesgo de reincidencia.

Después del lóbulo temporal, el lóbulo frontal es otro sitio de donde frecuentemente pueden originarse focos epileptogénicos. Estos generalmente se localizan en la porción medial o inferior del lóbulo, motivo por el cual es difícil de localizar con EEG con electrodos de superficie. Varios autores han mostrado que el PET es una buena herramienta de estudio para estos pacientes, para tratar de localizar el foco y para determinar la existencia de más de una zona con hipometabolismo, en cuyo caso aumenta la posibilidad de crisis post-operatorias ${ }^{(16,18)}$ (Figura 2).

\section{Enfermedad Cerebro Vascular}

La enfermedad cerebro vascular se caracteriza, como su nombre lo indica, por el daño al tejido cerebral secundario a un evento que altere su irrigación, que puede ser oclusivo o hemorrágico. Aproximadamente dos tercios son isquémicos y el $75 \%$ son primer episodio. Cuando se produce un infarto cere- bral, el metabolismo del territorio vascular afectado se ve comprometido rápidamente y subsecuentemente se produce un desacoplamiento entre la perfusión y el metabolismo en la periferia del infarto: la perfusión disminuye relativamente con relación al consumo de glucosa o de oxígeno; estos últimos no se ven afectados significativamente ("misery perfusion").

En un infarto cerebral pueden existir otras áreas con compromiso de la perfusión y el metabolismo de la glucosa, alejadas del territorio primariamente afectado. El ejemplo más conocido es la diasquisis cerebelosa cruzada, que consiste en hipometabolismo e hipoperfusión contralateral al territorio cortical afectado y ocurre dentro de los dos primeros meses siguientes del infarto. Se cree que es una manifestación secundaria a la interrupción del tracto cortico-ponto-cerebeloso (deaferentación). Se ha observado que esta manifestación es un signo de mal pronóstico en la recuperación de las funciones neurológicas de los pacientes con infarto cerebral, probablemente porque es proporcional a la extensión y gravedad de la lesión. Otras áreas que pueden presentar hipometabolismo alejados del territorio primario son el tálamo y el núcleo caudado ipsilateral a la zona dañada. En la etapa aguda (primeras 48 horas) los estudios de perfusión cerebral con SPECT presentan mayor sensibilidad que la tomografía computada para detectar infartos cerebrales, $79 \%$ versus $35 \%$ respectivamente, especialmente cuando se trata de infartos corticales (sensibilidad 93\%). Con el uso de radiofármacos para SPECT, especialmente Tc-99m HMPAO, es posible observar un fenómeno denominado "perfusión de lujo", donde el tamaño del infarto puede ser significativamente subestimado. Esto se puede evitar obteniendo las imágenes en las primeras 24 horas del evento.

Estudios con F-18 FDG PET han sido usados para evaluar la efectividad de varios tipos de tratamiento del infarto cerebral. Por ejemplo en trombolisis en la etapa aguda, el PET ha demostrado que tejido críticamente comprometido puede ser preservado mediante la reperfusión precoz y que grandes infartos pueden ser prevenidos mediante la trombolisis en etapa aguda, dejando áreas con perfusión disminuida pero viable ("misery perfusion") ${ }^{(16)}$.

\section{Hemorragia Intracraneal}

El uso de estudios de perfusión cerebral con SPECT han demostrado ser de utilidad en pacientes con hemorragia subaracnoídea, especialmente en la evaluación del vasoespasmo. En este contexto es útil el uso de la prueba con acetazolamida, que es un inhibidor de la anhidrasa carbónica. Cuando la concentración de $\mathrm{CO}_{2}$ aumenta, se produce vasodilatación cerebral y aumento del flujo regional. 


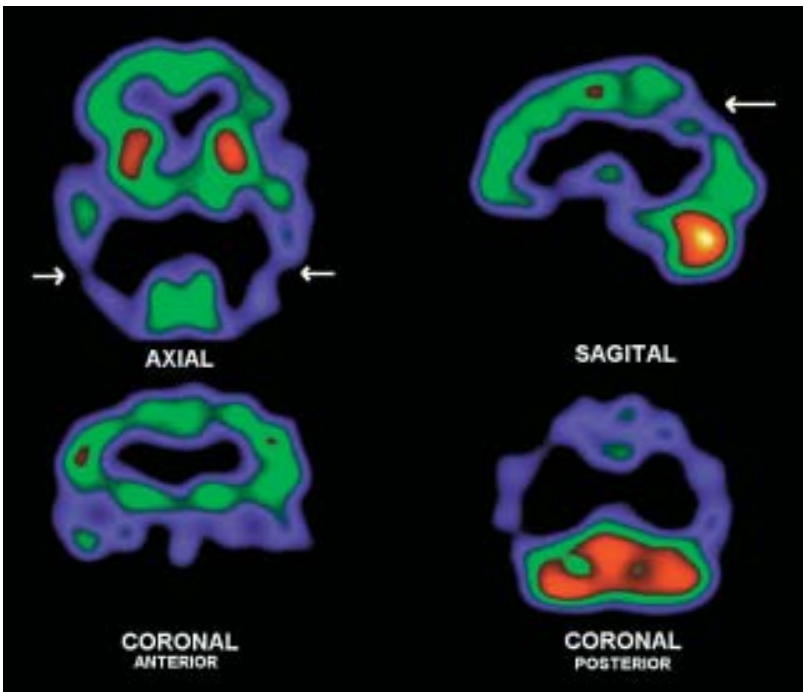

Figura 1. Enfermedad de Alzheimer. Antecedentes: Paciente de 68 años de sexo femenino, consulta por deterioro psico-orgánico de rápida evolución, caracterizado por trastornos de memoria, lenguaje (disminución y procacidad), comportamiento (inquietud permanente $y$ agresividad en el lenguaje). Solo es capaz de seguir órdenes muy simples. Hallazgos: Se observa marcada disminución global de la perfusión cortical, de predominio temporo-parietal bilateral (imagen axial, sagital [flechas] y coronal posterior) áreas azules; con relativa preservación de la corteza sensorio-motor (imagen coronal anterior). Los núcleos basales y estructuras infratentoriales se aprecian de perfusión conservada (cerebelo en tono más intenso).

Con esta prueba es posible medir la reserva vascular cerebral: en presencia de vasoespasmo, no se produce vasodilatación con la Acetazolamida, lo que se manifiesta en las imágenes como regiones hipoperfundidas especialmente en los territorios vasculares distales ${ }^{(16)}$.

\section{Isquemia Transitoria}

Es un área donde los estudios de perfusión con SPECT o metabólicos con PET podrían ser potencialmente muy importantes. Cuando la perfusión cerebral disminuye, la extracción de oxígeno aumenta en forma compensatoria ("misery perfusion"). En estados de isquemia crónicos, se produce vasodilatación máxima de los territorios afectados, lo que se denomina pérdida de la reserva vascular cerebral. El uso de la prueba con Acetazolamida con SPECT puede identificar aquellos con pérdida de la reserva vascular cerebral y en riesgo por lo tanto de desarrollar un evento isquémico.

Utilizando una combinación de radiotrazadores como $\mathrm{O}^{15} \mathrm{O}_{2}, \mathrm{O}^{15} \mathrm{CO}_{2}$ y $\mathrm{O}^{15} \mathrm{H}_{2} \mathrm{O}$, se puede medir con PET una serie de parámetros como volumen sanguíneo cerebral, extracción de oxígeno, metabolismo regional de oxígeno y flujo sanguíneo cerebral. De todos estos, la determinación de extracción de oxí-

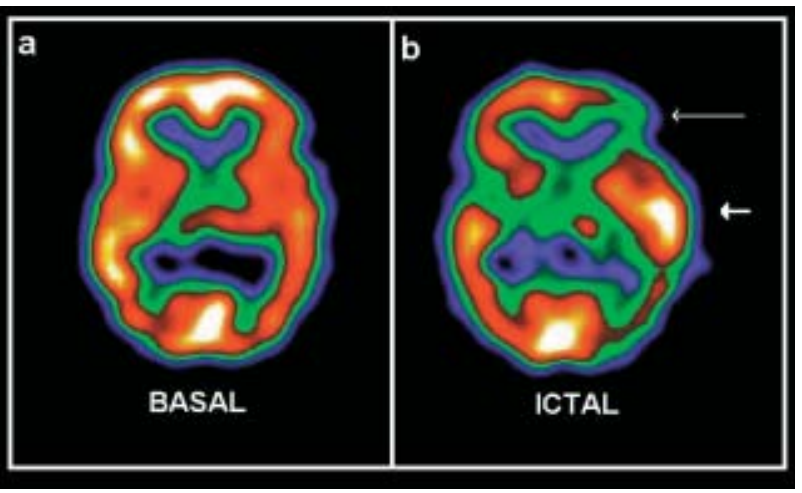

Figura 2. Epilepsia. Antecedentes: Paciente de 14 años de sexo masculino, con epilepsia parcial. Video EEG continuo no puede localizar foco epileptogénico, probablemente fronto-temporal izquierdo. Hallazgos: a.- Estudio basal muestra disminución de flujo, de lóbulo temporal izquierdo. b.- SPECT ictal muestra gran aumento de la perfusión en lóbulo temporal izquierdo especialmente en la región mesial que se extiende a la corteza lateral (flecha corta), con hipoperfusión relativa del polo anterior y región temporal anterior izquierda (flecha larga).

geno parece ser el método más confiable para determinar el compromiso de la circulación cerebral incipiente. En aquellos pacientes en que se identifica aumento de la extracción de oxígeno distal a una estenosis, tienen un riesgo más elevado de tener un evento isquémico en el futuro(16).

\section{Tumores Cerebrales}

Los tumores cerebrales representan el 5-9\% de todas las neoplasias y tienen una sobrevida promedio de un año. Los gliomas representan el $50 \%$ de todos los tumores intracraneales. Los estudios con PET pueden tener un rol importante en la evaluación y tratamiento de estos pacientes incluyendo la graduación del tumor, determinación del pronóstico y diferenciación de recurrencia versus radionecrosis.

En general el grado de hipermetabolismo del tumor se correlaciona directamente con su grado histológico. Al respecto, la forma más confiable de medir el hipermetabolismo es mediante un índice cuantitativo en comparación con el tejido cerebral sano contralateral. Una excepción a esta regla está dado por el astrocitoma pilocítico juvenil que es hipermetabólico a pesar de ser benigno histológicamente. Este hallazgo puede ser atribuido a una mayor expresión de transportadores de glucosa, más que un aumento del metabolismo propiamente tal. Hipometabolismo se asocia a la existencia de edema, transformación quística y necrosis. También puede haber diasquisis cerebelosa cruzada en tumores corticales y diasquisis cortical cruzada en tumores cerebelosos.

Estudios con F-18 FDG PET tienen valor pronóstico, se ha observado que este método puede 
predecir mejor el pronóstico de los pacientes con tumores cerebrales que los métodos de imágenes estructurales. EI PET puede estimar mejor el grado de malignidad y se ha observado que aquellos pacientes que tienen tumores hipermetabólicos tienen una sobrevida claramente diferente (promedio 7-11 meses) de aquellos con tumores hipometabólicos (promedio 33 meses, rango 1-más de 7 años).

Con relación a la radionecrosis, el uso de F-18 FDG PET puede ser de utilidad: las áreas de necrosis generalmente son hipometabólicas y las de recurrencia presentan aumento del metabolismo de la glucosa. La sensibilidad y especificidad de éste método es de alrededor de $86 \%$ y $56 \%$ respectivamente $^{(4)}$.

\section{Trastornos del Animo}

En los pacientes con depresión generalmente se produce un trastorno global del metabolismo de la glucosa, manifestado por disminución del consumo de la glucosa difusamente en la corteza cerebral e incluso en algunas estructuras grises subcorticales. Se ha observado que en los pacientes con formas familiares de depresión (ya sea uni o bipolar) presentan hipometabolismo de un área localizada en la corteza prefrontal ventral a la rodilla del cuerpo calloso. En aquellos pacientes que presentan síntomas ansiosos asociados con la depresión, presentan cambios metabólicos específicos: aumento de la actividad parahipocampal derecha y de la circunvolución cingulada anterior izquierda y disminución en el cerebelo, circunvolución fusiforme, temporal superior, angular e ínsula del lado izquierdo(4).

\section{Esquizofrenia}

Es una enfermedad que ha sido extensa e intensamente investigada, a pesar de lo cual muy poco se conoce respecto de su etiopatogenia y fisiopatología. Se cree que la disfunción del lóbulo frontal explica en parte la sintomatología de estos pacientes. Al respecto se ha observado una correlación entre el hipometabolismo de este lóbulo con la presencia de síntomas negativos. Se ha propuesto la existencia de tres subgrupos de esquizofrénicos ${ }^{(19)}$, cada uno con un síndrome característico y un patrón metabólico cerebral diferente:

a) Pacientes con un síndrome de disminución de la actividad psicomotora y de lenguaje que se acompaña de disminución del metabolismo de la corteza prefrontal dorso-lateral.

b) Pacientes con un síndrome desorganizativo, con incapacidad de inhibir respuestas inapropiadas. Esto se acompaña de incremento del metabolismo de la glucosa en la circunvolución cingulada anterior derecha.

c) Pacientes con síndrome de distorsión de la realidad con aumento del metabolismo en el lóbulo temporal medial.

Los estudios con PET también han mostrado cambios en el metabolismo cerebral de los esquizofrénicos tratados con neurolépticos. En aquellos pacientes que responden a la terapia con haloperidol se ha observado una mejoría del metabolismo de la glucosa, mientras que los no respondedores muestran un empeoramiento del metabolismo frontal ${ }^{4)}$.

\section{Trastorno Obsesivo Compulsivo}

En este trastorno se ha observado un incremento del metabolismo de la glucosa en la corteza órbitofrontal y en el núcleo caudado. También puede observarse aumento del metabolismo de la porción anterior de la circunvolución cingulada(4)

\section{Alcoholismo}

Estudios con PET en alcohólicos crónicos han demostrado una disminución difusa del metabolismo cerebral en la corteza y también en estructuras grises subcorticales. Puede haber mayor compromiso de algunas áreas como la región frontal u occipital y en los pacientes con atrofia cerebelosa, se puede ver además disminución del metabolismo en la porción superior del vermis. Al parecer las mujeres demuestran menores cambios en el metabolismo comparado con los hombres. Durante los períodos de abstinencia, se observa una mejoría parcial del metabolismo cerebral (aunque pueden permanecer algunas áreas frontales o temporales hipometabólicas) que es más pronunciado en los primeros 13-30 días. Estas mejorías se correlacionan positivamente con mejorías en las pruebas neuropsicológicas cognitivas y de funciones ejecutivas. Los pacientes que recaen, presentan empeoramiento del metabolismo.

El efecto agudo incluye disminución del metabolismo en corteza occipital, prefrontal y cerebelosa, más pronunciado en los pacientes alcohólicos que en los controles. Estas áreas coinciden con las regiones donde mayor cantidad de receptores de benzodiazepinas existe; drogas que se usan en el tratamiento del síndrome de deprivación alcohólica ${ }^{(4)}$.

\section{Cocaína}

El abuso de cocaína ha aumentado dramáticamente en los últimos años y ha llegado a proporciones epidémicas. Durante la exposición aguda a la sustancia, se produce una disminución global del metabolismo en estructuras corticales y subcorticales, en forma proporcional a la sensación de euforia producida. Durante el período de detoxificación se ha observado regresión de algunas de las alteraciones metabólicas, en función del tiempo transcurrido (hasta dos meses). También se ha descrito el patrón metabólico que se asocia al síndrome de deprivación que consiste en hipermetabolismo de la región órbito- 
frotal y de los ganglios basales, este fenómeno se observó una semana después de la última exposición, pero no se observó luego de un mes de abstinencia ${ }^{(4)}$.

\section{Traumatismo Encéfalo Craneano}

En Chile la cuarta causa de muerte la producen los accidentes traumáticos y alrededor del $50 \%$ de los casos fatales son por traumatismo encéfalo craneano $(T E C)^{(20)}$. En la evaluación aguda del TEC las imágenes de perfusión cerebral con SPECT tienen un valor pronóstico, en general pacientes con mayor número o extensión de lesiones de perfusión en relación a las lesiones demostradas con resonancia magnética (RM) o tomografía axial computada (TAC), tienen peor pronóstico, mientras que aquellos en los cuales el SPECT inicial es normal, el pronóstico es excelente. En la etapa crónica numerosos autores han demostrado que los estudios funcionales (SPECT o PET) muestran mayor número de alteraciones que las imágenes estructurales (TAC o RM) y que las anormalidades en las pruebas neuropsicológicas tienen buena correlación con las primeras. Más aún, en pacientes en rehabilitación post-TEC se ha demostrado que las mejorías observadas en las pruebas neuropsicológicas se correlacionan con mejoría en la perfusión cerebral medida con SPECT ${ }^{(21)}$.

\section{Conclusión}

El aumento en la disponibilidad de técnicas como F-18-FDG PET y el desarrollo de nuevos radiofármacos, especialmente radioligandos para neurorreceptores, cada vez más específicos y marcados con isótopos de mayor disponibilidad, permitirán incrementar nuestro conocimiento sobre los mecanismos subyacentes en numerosas patologías neuro-psiquiátricas y de esta forma enfocar los esfuerzos terapéuticos. En el futuro deberían desarrollarse nuevas técnicas para el estudio dinámico de los cambios neuroquímicos in vivo.

\section{Bibliografía}

1. Food and Drug Administration Modernization Act 121. Positron Emission Tomography, 1997.

2. Chawluk JB, Alavi A, Dann R, et al: Positron emission tomography in aging and dementia: Effect of cerebral atrophy. J Nucl Med 28:431-437, 1987

3. Weiss D, Sodee E, Alavi A, et al: Effects of normal aging on whole brain and regional glucose metabolism as assessed by $\mathrm{F}-18$ positron emission tomography. $\mathrm{J} \mathrm{Nucl}$ Med 31:771, 1990

4. Newberg A, Alavi A \& Reivich M: Determination of Regional Cerebral Function with FDG-PET Imaging in Neuropsychiatric Disorders. Semin Nucl Med 32 (1): 13-34, 2002

5. Yoshii F, Barker WW, Chang JY et al: Sensitivity of cerebral glucose metabolism to age, gender, brain volume, brain atrophy and cerebrovascular risk factors. J Cerebr Blood Flow Metab 8:654-661, 1988

6. McKhann G, Drachman D, Folstein M, et al: Clinical Diagnosis of Alzheimer's Disease: Report of the NINCDS-ADRDA Work group under the auspices of Department of Health and Human Services Task Force on Alzheimer's Disease. Neurology 34:939-944, 1984

7. Bonte FJ, Hom J, Tinter R, et al: Single photon tomography in Alzheimer's disease and the dementias. Semin Nucl Med 20:342-352,1990

8. Mazziota JC, Franckowiak RSJ, Phelps ME: The use of positron emission tomography in the clinical assessment of dementia. Semin Nucl Med 22:232-246, 1992

9. Ishii K, Sasaki M, Sakamoto S, et al: Tc-99m ethyl cysteine dimer SPECT and 2-[F-18] FDG PET in Alzheimer's Disease: Comparison of perfusion and metabolic patterns. Clin Nucl Med 24:572-575, 1999

10. Bonte FJ, Weiner MF, Bigio EH, et al: Brain blood flow in the dementias: SPECT with histopathologic correlation in 54 patients. Radiology 202:793-797,1997

11. Newberg A, Alavi A, Clark C: The metabolic imaging severity rating scale (MISRS) in Alzheimer's disease: Comparison with quantitative data. Eur J Nucl Med 25:1068, 1998

12. Jagust WJ, Haan MN, Reed BR, et al: Brain perfusion imaging predicts survival in Alzheimer's disease. Neurology 51:1009-1013, 1998

13. Donnenmiller E, Heilmann J, Wenning GK, et al: Brain perfusion scintigraphy with 99mTc-HMPAO or 99mTcECD and 123I-beta-CIT single-photon emission tomography in dementia of the Alzheimer-type and diffuse Lewy body disease. Eur J Nucl Med 24:320325, 1997

14. Ishii K, Yamaji S, Kitangaki H, et al: Regional cerebral blood flow difference between dementia with Lewy bodies and AD. Neurology 53:413-416, 1999

15. Ishii K, Imamura T, Sasaki M, et al: Regional cerebral glucose metabolism in dementia with Lewy bodies disease and Alzheimer's disease. Neurology 51:125130,1998

16. Van Heertum RL, Drocea C, Ichse M, et al: Single photon emission CT and positron emission tomography in the evaluation of neurologic disease. Radiol Clin North Am 39:1007-34, 2001

17. Mazziota JC: Huntington's disease: Studies with structural imaging techniques and positron emission tomography. Semin Neurol 9:360-369,1989

18. Rowe C: Nuclear medicine in the management of a patient with epilepsy in Murray IPC, Ell PJ (eds): Nuclear Medicine in clinical diagnosis and treatment. Edingburgh, Churchill Livingstone.1998, pp 651-666

19. Liddle PF, Friston KJ, Fritch CD, et al: Patterns of cerebral blood flow in schizophrenia. $\mathrm{Br} \mathrm{J}$ Psychiatr 160:179-186,1992

20. Mendez J: Traumatismo Encéfalo Craneano. Revisiones de temas neuroquirúrgicos, http:// escuela.med.puc.cl/Departamentos/neurocirugia/ publtec.htm

21. Laatsch L, Pavel D, Jobe $T$, et al: Incorporation of SPECT imaging in a longitudinal cognitive rehabilitation therapy programme. Brain Inj 13:555-570, 1999 